Computer Optics and Nanophotonics

\title{
HYPERSPECTRAL GAS ANALYZER FOR MONITORING OF OIL AND GAS PIPELINES
}

\author{
A.D. Golovin, A.V. Demin \\ ITMO University (Saint Petersburg National Research University of Information Technologies, \\ Mechanics and Optics), Saint Petersburg, Russia, \\ JSC "LOMO", Saint Petersburg, Russia
}

\begin{abstract}
For the detection and identification of hydrocarbons and other volatile organic compounds (VOCs) conceptual design of a compact hyperspectral instrument with high spatial and spectral resolution was developed. This paper presents the calculation of the optical thickness of the medium ranges and an evaluation of the measurement uncertainty for the different spectral windows of atmospheric transparency. The optical system, the analytical design and calculation of energy, and the algorithm for selecting optimal parameters for the system were developed on the framework of the Offner scheme.
\end{abstract}

Keywords: ecological monitoring, detection of VOCs, hyperspectrometer Offner, UAV, signal/noise ratio, optical depth.

Citation: Golovin AD, Demin AV. Hyperspectral gas analyzer for monitoring of oil and gas pipelines. CEUR Workshop Proceedings, 2016; 1638: 166-172. DOI: 10.18287/1613-0073-2016-1638-166-172

\section{Introduction}

The extraction and transportation of hydrocarbons is now the dominant sector of the Russian economy. There are now more than 930 thousand $\mathrm{km}$ of gas and oil pipelines in Russia. In light of the consequences of ecological problems, the loss of raw materials due to large and small leaks, and increased incidences of illegal tapping of gas and oil pipelines, there is an increased need to secure monitoring of the infrastructure and complexes to control the proper technical condition of each portion of the system [1]. The Analysis of the known methods and means for monitoring gas and oil pipelines in the optical range from the aircraft (helicopter) showed that they all carry a high probability of errors in the identification of breakdown. The optoelectronic equipment designed for these systems does not provide a solution to all problems. In particular, it has problems in small leak detection and the identification of gases with in-time thematic maps building. In addition, monitoring systems are usually purchased in foreign countries and come at a heavy cost [2-5]. 
There is a necessity to create and develop small and compact monitoring systems for use in the field and in remote areas with a broad scope and application versatility, enabling to analyze and identify hydrocarbons and other volatile organic compounds (VOCs) based upon their detailed spectral characteristics.

The uniqueness of the hyperspectral system is it opportunities to fix the light at hundreds of very narrow spectral bands, which make it possible to assess the physicochemical properties of the objects. To collect the "hypercube" (cubic model image) the investigated scene is scanned along the direction of motion of the platform, at the same time collecting the second dimension of the detector's entire spectral information. The main advantage of this mode is a synchronous linear dispersement while collecting the spectrum without the need for post-processing [6-9].

An Unmanned Aerial Vehicle (UAV) is equipped hyperspectral module, able to collect the spectral and topological data in a high-resolution area of interest without significant costs (Figure 1).

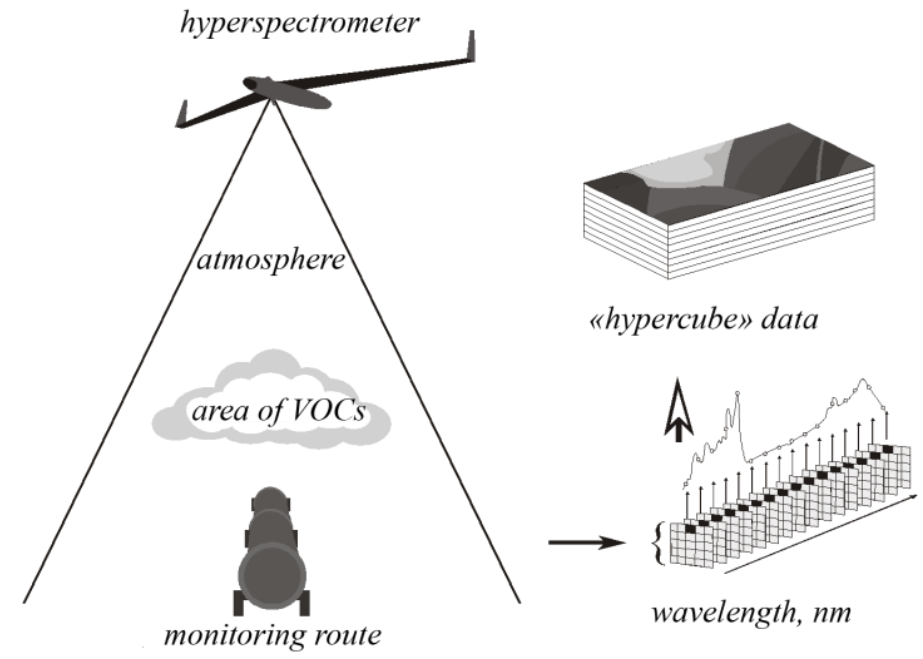

Fig. 1. The scheme for obtaining hyperspectral data

In this paper, we propose a method and a means of monitoring gas and oil pipelines on board aircraft, including UAVs. As a method for assessing the state of the gas and oil pipeline proposed hyperspectral analysis, as well as a means of compact hyperspectrometer based on the Offner schemes.

Figure 2 shows a block diagram of compact hyperspectrometer for monitoring of oil and gas pipelines in the spectral ranges $(1-2.5 ; 3-5 ; 8-11)$ microns. The main functional blocks - multispectral objective $\left(2 \omega=8.5^{\circ} ; 1: 1.5\right)$; spectrum analyzer on the framework of the Offner scheme; photodetectors block. 


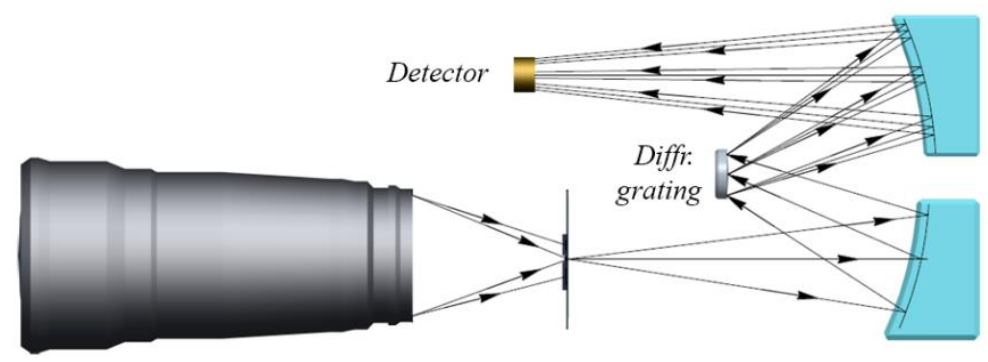

Fig. 2. Structural diagram of hyperspectrometer.

\section{The calculation of signal/noise ratio}

To estimate the optical depth of the medium range, carry out calculation of signal/noise ratio in the obtained spectrum of the registration of hyperspectral images of spectral intervals corresponding to the windows of atmospheric transparency. In this case, we assume that the aperture size of the input window, the spectral and spatial resolution for devices on different spectral ranges, remain constant.

Consider the spectral range of 8 to 11 microns.

The maximum stream that may occur in the study of underlying surface of the Earth may be considered stream emitted by a black body (with emissivity equal to one), having a temperature of about $320 \mathrm{~K}$. The integral stream of a black body with a temperature that emits in the spectral range of 8-11 microns is:

$B_{8-11}=4.4 \cdot 10^{-3} \mathrm{Watt} / \mathrm{cm}^{2} \mathrm{sr}$.

The solid angle in which the individual pixel of matrix detectors receives emission is: $\Omega=S_{E} / h^{2} \cong 2.21 \cdot 10^{-7} \mathrm{sr}$,

where $S_{E}$ - area of Earth, the size of which is covered by one pixel detector $47 \mathrm{~mm} \times 47 \mathrm{~mm}), \mathrm{h}-$ distance to device $100 \mathrm{~m}$.

If we accept that the area the input window of device is a $S_{H}=12.57 \mathrm{~cm}^{2}$, the total transmission scheme can be accepted as $\tau=0.7$, the integrated stream on detector received from a black body at $\grave{O}=320 \hat{E}$ :

$\Phi=B_{8-11} \Omega S_{H} \tau=4.4 \cdot 10^{-3} \cdot 2.21 \cdot 10^{-7} \cdot 12.57 \cdot 0,7=8.56 \cdot 10^{-9} \mathrm{Watt}$.

Assuming that the energy of a quantum for a wavelength 10 microns is $\AA_{q t 10}=\frac{\mathrm{h} \cdot \mathrm{c}}{\lambda}=2 \cdot 10^{-20}$ Joule, the number of quanta per one pixel of the detector integrated flux in the spectral range of 8-11 microns:

$N_{q t}=4.28 \cdot 10^{11}$ quantum per second.

The hyperspectral device has a spatial encoding wavelength. Coming in on the grating integrated emission is divided by the number required for the registration of the spectral ranges, and each of these divided emission parts registered individual pixels (really, 
mostly by one pixel accounts for only part of the small stream as a spectral interval must be recorded, at least, two pixels). Consequently, in the best case, one pixel comes (in this range, to a first approximation, we can assume that the energy is distributed uniformly over the entire spectral range) $N_{\text {qt. peak }}=N_{q t} / \grave{I}$ where $\grave{I}$ - number of spectral ranges. If $\grave{I}=300$, then $N_{\text {qt. peak }}=2.14 \cdot 10^{9}$ quantum per second. For the chosen system of registration and selected parameters of the device, the averaging time is about $10 \mathrm{~ms}$, with a conversion efficiency of 0.6.

The value of the signal is $S_{g r}=8.58 \cdot 10^{6}$ electrons, the value of noise is: $N_{\text {noise }}=1000$ electrons.

$\mathrm{S} / \mathrm{N}$ ratio in the range of the maximum signal is $\chi \cong 8580 \cong 79 \mathrm{~dB}$.

Consider the spectral range of 3 to 5 micrometers under the same conditions:

$B_{3-5}=3.5 \cdot 10^{-4} \mathrm{Watt} / \mathrm{cm}^{2} \mathrm{sr}$;

$\Phi=B_{3-5} \Omega S_{H} \tau=3.5 \cdot 10^{-4} \cdot 2.21 \cdot 10^{-7} \cdot 12.57 \cdot 0.7=6.8 \cdot 10^{-10}$ Watt $;$

$\AA_{\text {qt } 4}=5 \cdot 10^{-20}$ Joule ;

$N_{q t}=1.36 \cdot 10^{10}$ quantum per second.

$N_{\text {qt. peak }}=4.53 \cdot 10^{7}$ quantum per second.

The hyperspectral device has $S_{g r}=4.53 \cdot 10^{7} \cdot 0.01 \cdot 0.6=2.72 \cdot 10^{5}$ electrons with the noise of $N_{\text {noise }}=1000$ electrons.

$\mathrm{S} / \mathrm{N}$ ratio in the range of the maximum signal is $\chi \cong 272 \cong 49 d B$.

Other spectral ranges are calculated, substantially, in the same way, but in place of own blackbody radiation at a certain temperature, the integral of the reflected solar energy is used with maximum albedo of 0.7 .

Consider the spectral range of 1-2.5 microns, wherein assume that the albedo for maximum signal is $\alpha=0.7$ :

$B_{1-2.5}=5.7 \cdot 10^{-3} \mathrm{Watt} / \mathrm{cm}^{2} \mathrm{sr}$;

$\Phi=B_{1-2,5} \Omega S_{H} \tau=5.7 \cdot 10^{-3} \cdot 2.21 \cdot 10^{-7} \cdot 12.57 \cdot 0.7 \cdot 0.7=7.76 \cdot 10^{-9}$ Watt $;$

$\AA_{\text {qt } 2}=10^{-19}$ Joule ;

$N_{q t}=7.76 \cdot 10^{10}$ quantum per second;

$N_{\text {qt. peak }}=2.6 \cdot 10^{8}$ quantum per second.

Assuming the conversion efficiency of 0.6 , signal acquisition time $10 \mathrm{~ms}$, the value of the signal is $S_{g r}=1.56 \cdot 10^{6}$ electrons, detector noise is 1000 electrons, $\mathrm{S} / \mathrm{N}$ ratio is equal to $\chi \cong 1560 \cong 64 d B$. 


\section{The calculation range of optical depth}

In remote monitoring, the obtained spectrum area containing volatile organic compounds (VOCs), allows for the simplification allocation algorithm echo. Its power is determined by the following expression relative to an emission wavelength $\lambda_{i}$ [9]:

$$
P\left(\lambda_{i}\right)=\tilde{K}\left(\lambda_{i}\right) P_{0}\left(\lambda_{i}\right) \exp \left\{-2 \sum_{j}^{k}\left(\int_{0}^{L} N_{j}(z) \sigma_{r j}\left(\lambda_{i}, z\right) d z\right)-2 \int_{0}^{L} \varepsilon\left(\lambda_{i}, z\right) d z\right\},
$$

where $P_{0}$-power emitter; $L$-distance to area of VOCs; $\sigma\left(\lambda_{i}, z\right)$-an indicator of scattering; $\varepsilon\left(\lambda_{i}, z\right)$ - an indicator of atmospheric attenuation; $K\left(\lambda_{i}, z\right)-$ the number of gas components; $N(z)$ - the concentration of harmful compounds. This ratio can be used to determine the threshold characteristics of the spectrum, considering required amount of signal/noise ratio for the detector. The transmission coefficient of VOC area is $\tau_{V O C}=\exp \left(-D_{V O C}\right)$ and for the scene is $\tau_{s c}=\exp \left(-D_{s c}\right)$, where $D$ - optical depth.

The observed spectrum area of VOCs at a specified wave number can be expressed as the following expression:

$\Upsilon(v)=\Phi_{0} e^{-D_{s c}} e^{-D_{v o c}}+P_{1}\left(1-e^{-D_{v o c}}\right) e^{-D_{s c}}+P_{2}\left(1-e^{-D_{s c}}\right)$,

where: $\Phi_{0}-$ the emission spectrum of the components Earth surface; $P_{1}\left(v, T_{1}\right), P_{2}\left(v, T_{2}\right)$ - Planck function with temperatures $T_{1}$ (area of VOCs) and $T_{2}$ (researched scene).

At a concentration of VOCs is equal to zero ( $\left.D_{1}=0\right)$, the spectral component is defined as:

$$
\Upsilon_{0}(v)=\Phi_{0} e^{-D_{S C}}+P_{2}\left(1-e^{-D_{S C}}\right),
$$

where $\Phi_{0}$ may be represented as:

$$
\Phi_{0}=\frac{\Upsilon_{0}-P_{2}\left(1-e^{-D_{S C}}\right)}{e^{-D_{S C}}}
$$

On the basis of the relations (4) and (2) transmission coefficient of VOCs area can be defined as:

$$
\tau_{V O C}(v)=\exp \left(-D_{V O C}\right)=\frac{\Upsilon-P_{2}+\Delta P e^{-D_{S C}}}{\Upsilon-P_{2}+\Delta P e^{-D_{S C}}}
$$

where $\Delta P=P_{1}-P_{2}$.

This expression is defined for temperatures $T_{1}, T_{2}$ and the height of area of VOCs and it can be simplified by adopting $T_{1} \approx T_{2}$ (which is typical for remote monitoring in field conditions [10]):

$$
\tau_{V O C}(v)=\exp \left(-D_{V O C}\right)=\frac{\Upsilon(v)-P(v, T)}{\Upsilon_{0}(v)-P(v, T)} \text {. }
$$

A mathematical condition for the spectral observation area of VOCs is: 


$$
\Delta \Upsilon(v, T)=\left[B_{0}(v)-P(v, T)\right] \neq 0
$$

Notice that when $\Delta \Upsilon(v, T)>0$, the absorption spectrum is recorded, while a negative value of $\Delta \Upsilon$ records VOCs emission spectrum area. Knowing the value of the observed spectrum, it is possible to determine the minimum concentration of VOCs, which the device can identify.

By adopting observed spectrum of positive, signal/noise ratio for the correlated spectrum $\Delta \Upsilon(v, T)$ equals $\chi(v)=\Delta \Upsilon(v) / \delta \Upsilon$, where $\delta \Upsilon-R m s$ noise. Hence, a minimum concentration recorded by the instrument, according to the law of Bouguer - Lambert - Beer law [11]:

$$
\tau_{\min }=\exp \left(-D_{\min }\right)=\frac{\Upsilon_{0}(v)-\delta \Upsilon-P(v, T)}{\Upsilon_{0}(v)-P(v, T)}=\left(1-\frac{1}{\chi}\right)
$$

From this expression, we can determine the minimum optical depth cloud of VOCs, identifiable by dint of hyperspectral device:

$D_{\min }=-\ln (1-1 / \chi)$

In the same way we obtain the following mathematical calculations for determine the maximum detectable optical depth cloud of VOCs:

$D_{\max }=\ln \left(\frac{\Upsilon_{0}(v)-[P(v, T)-\delta \Upsilon]}{\Upsilon_{0}(v)-P(v, T)}\right)=\ln (\chi)$.

To calculate the relative error of measurement device, accept that (that is caused high values of FPA noise in the infrared range of the spectrum [12]). Thus, the relative error in determining optical depth can be found from the expression:

$\theta=\frac{\delta D}{D}=\frac{1}{D} \sqrt{\left(\frac{\partial D}{\partial \Upsilon} \delta \Upsilon\right)^{2}+\left(\frac{\partial D}{\partial \Upsilon_{0}} \delta \Upsilon_{0}\right)^{2}}=\frac{1}{\chi D} \sqrt{1+e^{2 D}}$.

The calculations of the minimum and maximum values determined by the device, the optical depth and the relative errors of measurement based on the calculated signal/noise ratio and the formulas (9), (10), (11) for the three spectral bands corresponding to the windows of atmospheric transparency. The calculation results are shown in the table:

Table 1. The results of calculation of optical depth and relative errors for different spectral ranges

\begin{tabular}{cccc}
\hline Calculated parameters & \multicolumn{3}{c}{ Spectral ranges, micron } \\
\cline { 2 - 4 }$\chi$ - the signal/noise ratio & $8-11$ & $3-5$ & $1-2,5$ \\
$D_{\min }$ - the minimal optical depth & $1.17 \cdot 10^{-4}$ & $3.6 \cdot 10^{-3}$ & 1560 \\
$D_{\max }$ - the maximal optical depth & 9.06 & 5.61 & 7.35 \\
$\theta_{\min }$ - the rel. error for opt. min. & 141 & 143 & 142 \\
$\quad$ depth, \% & & & 14 \\
$\theta_{\max }$ - the rel. error for opt. max \\
depth, \%
\end{tabular}




\section{Conclusion}

Preliminary experimental studies in the laboratory have confirmed the chosen solutions to be correct. The hyperspectrometer conceptual project to assess oil and gas pipelines from aircraft (helicopter) was developed as a result of theoretical and experimental research. The results show the advisability of the development of a prototype and of full-scale field research.

\section{References}

1. Palatov YA, Knyazev NA et al. Monitoring of emergencies, fires and industrial emissions with the help of space vehicles observations, 19-21 June 2006, SSTC RF ISTC "Karpov", M .: 2006 .

2. Novgorodskaya AV. Review of the methods of contactless remote detection and identification of hazardous substances. Engineering Journal: Science and Innovation, $2013 ; 8$.

3. Voronov V. Complex system monitoring facilities of JSC "Gazprom" with the help of UAVs. "NefteGazAeroKosmos" FSPC. URL: http://uav.ru/articles/KSKM.pdf (reference date 18.03.2016).

4. Hrenov NN. Fundamentals of complex diagnostics northern pipelines. Aerospace Methods and materials processing surveys. M.: Gasoil press, 2003; 352 p.

5. Golovin AD, Demin AV. Simulation model of a multichannel Offner hyperspectrometer. Computer Optics, 2015; 39(4): 521-528. DOI: 10.18287/0134-2452-2015-39-4-521-528.

6. Kazanskiy NL, Kharitonov SI, Doskolovich LL, Pavelyev AV. Modeling the performance of a spaceborne hyperspectrometer based on the Offner scheme. Computer Optics, 2015; 39(1): 70-76. DOI: 10.18287/0134-2452-2015-39-1-70-76.

7. Golovin AD, Demin AV. Compact hyperspectrometer for visible and Swir wavelength range. Journal of Instrument Engineering, 2015; 58(11): 869-875. DOI: 10.17586/00213454-2015-58-11-869-873.

8. Lyalko VI, Fedorov OD, Popov MO et al. Multi-spectral methods of remote sensing of the Earth in the wildlife management problems. Naukova Dumka, 2006; 357 p.

9. Kozintsev VI, Orlov VM, Belov ML et al. Optoelectronic environmental monitoring of the environment: Textbook. manual for schools. Ser. Electronics. Ed. Rozhdestvina VN. M.: Publishing House of the MSTU Bauman, 2002; 528 p.

10. Welton EJ, Campbell JR, Berkoff TA et al. The Micro-pulse Lidar Network (MPL-Net) Lidar Remote Sensing in Atmospheric and Earth Sciences: Reviewed and revised papers at the twenty-first Int. Laser Radar Conference (ILRC21). Part I. Quebec. Canada, 8-12 July 2002.

11. Morozov AN, Svetlichniy SI. Basics of Fourier spectroradiometer. Ed. Vasiliev GK. Inst. energy resou. chemical problems. Physics. Moscow: Nauka, 2006; 275 p.

12. Zarco-Tejada PJ, Gonzalez-Dugo V, Berni JAJ. Fluorescence, temperature and narrowband indices acquired from a UAV platform for water stress using a micro-hyperspectral images and a thermal camera. Remote Sens. Environ, 2012; 117: 322-337. 